\section{Anarchy in the beehive}

SIR - Worker bees in eusocial Hymenoptera colonies often have functional ovaries which can produce fertile male eggs'. The sons of a worker's 'super sister' are only $3 / 4$ as much related to her as her own sons, and only $1 / 2$ as much related to her as sons of the queen. This leads to the possibility of intense competition among workers for the production of
(3) workers carrying queen-laid drone eggs through the queen excluder.

A candidate colony was reported from Ipswich, Queensland in late winter, before swarming season. The colony was normal in every respect except that most drone-sized cells $(>100)$ above the queen excluder contained brood. No worker brood was present above the queen

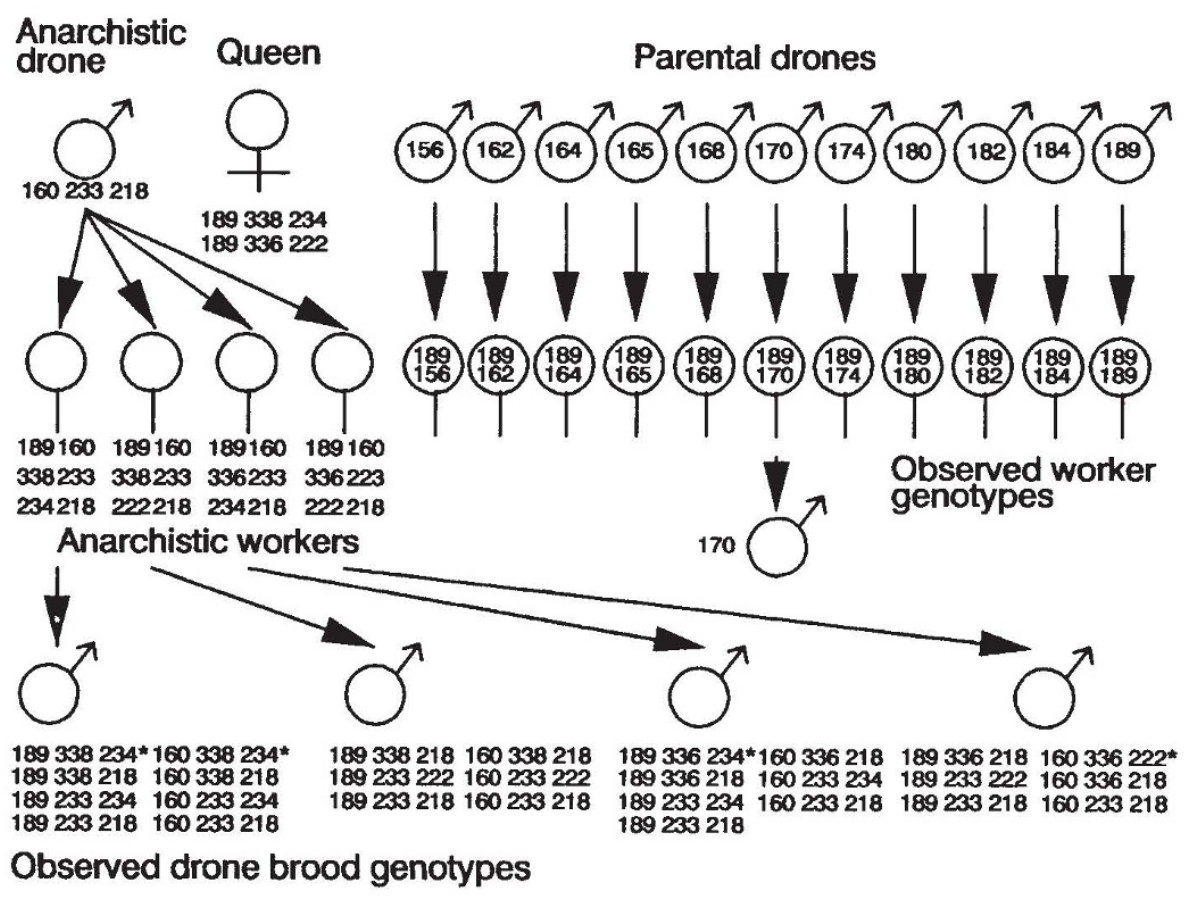

Pedigree of a honeybee colony determined from three microsatellite loci. Drones marked with an asterisk are unique to that family, and indicate that at least three workers laid eggs.

males $^{2}$. In many species, however, worker reproduction is extremely rare ${ }^{3,4}$, probably because it is suppressed by a mutual 'policing' behaviour in which workers prevent others from laying eggs ${ }^{5,6}$. In honeybees there is good evidence that the mechanism of worker policing involves the marking of queen-laid eggs by a pheromone. In queen-right colonies, workers can identify worker-laid eggs by the lack of this putative pheromone, and eat them ${ }^{7}$. This system works very well most of the time, but is open to the invasion of 'anarchistic' behaviour. Here we report a colony where workers of a single patriline 'cheated' to produce many of the colony's male offspring.

Occasionally colonies are seen where most drones are likely to be offspring of workers, not the queen ${ }^{4}$. We asked beekeepers to report colonies where drone pupae were present above a queen exclud$\mathrm{er}$ in otherwise normal colonies. Because a queen cannot pass through a queen excluder, drone pupae above it can only result from: (1) a second queen above the queen excluder; (2) laying worker(s); or excluder. We obtained samples of worker brood from below the queen excluder, and adult workers and 49 drone pupae from above the excluder. Drone pupae were only present in drone-sized cells. We extracted DNA from these samples and analysed it for variation at three microsatellite $\operatorname{loci}^{8}$ to determine the paternity and maternity of each bee. Analysis of 128 adult and 74 pupal workers with microsatellite A107 revealed that every worker carried a 189 base-pair (bp) allele. Therefore the single queen heading allele. Twelve A107 alleles were present in the worker progeny indicating that the queen had mated with at least 12 drones. Examination of 12 workers with additional microsatellites A14 and A76 revealed that the queen was heterozygous $338 / 336$ and 234/222 for these loci, as all workers carried at least one of these alleles.

From the distribution of microsatellite alleles among the drone pupae, the most parsimonious hypothesis is that they are offspring of at least three workers sired by the same drone of genotype 160-233-218. the colony was homozygous for the $189 \mathrm{bp}$
One additional drone was descended from a worker of another patriline marked by the 170 allele at locus A107. It is overwhelmingly likely that the remaining 48 drones were derived from workers of a single patriline because only one nonqueen allele was found at each microsatellite locus in all drones examined.

It is almost impossible for our sample to have contained any drones laid by the queen. In 42 cases this possibility can be definitely excluded because these drones did not carry a queen allele at one or more loci. In the other six cases, it is possible but unlikely, as workers would have to have moved queen-laid eggs through the queen excluder ${ }^{7}$.

We conclude that anarchistic behaviour is genetically determined, that one of the males mating this queen passed an ability to his worker offspring to evade the policing mechanism. If queen-laid eggs are protected from policing by a queen-derived pheromone ${ }^{5,7}$, then it is likely that several bees of this patriline were able both to develop ovaries and to produce an acceptably queen-like pheromone that protected their eggs. It seems likely that workers of this patriline were more queen-like ${ }^{9}$ than normal bees. There is large genetic variance in the speed at which individuals develop ovaries and the probability that they will become 'false queens' in queenless colonies ${ }^{4}$.

This anarchistic behaviour could rapidly spread in honey bee populations. The fitness of these bees is higher than that of workers that cannot evade policing. Selection on workers to police this behaviour is expected to be strong given the number of times the queen mates ${ }^{2}$. These results support the worker policing hypothesis, while demonstrating a previously unrecognized dynamic equilibrium between reproductive conflict and cooperation.

Benjamin P. Oldroyd

Adam J. Smolenski

Jean-Marie Cornuet

Ross H. Crozler

School of Genetics and

Human Variation,

La Trobe University,

Bundoora, Victoria 3083,

Australia

J.-M. C. is on sabbatical from Laboratoire de Neurobiologie Comparée des Invertébrés, INRA CNRS URA 11290, La Guyonnerie, BP 23, 91440 Bures-sur-Yvette, France.

1. Oster, G. F. \& Wilson, E. O. Caste and Ecology in the Social Insects (Princeton University Press, 1978).

2. Ratnieks, F. L. W. Am. Nat. 132, 217-236 (1988)

3. Visscher, P. K. Behav. Ecol. Sociobiol. 25, 247-254 (1989).

4. Page, R. E. \& Erickson, E. H. Behav. Ecol. Sociobiol. 23, 117-126 (1988)

5. Ratnieks, F. L. W. \& Visscher, P. K Nature 342, 796-797 (1989).

6. Starr, C. K. in Sperm Competition and the Evolution of Animal Mating Systems (ed. Smith, R.L.) 428-459 (Academic, Orlando, 1984).

7. Ratnieks, F. L. W. Behav. Ecol. Sociobiol. 32, 191-198 (1993).

8. Estoup. A.,Solignac, M. \& Cornuet, J.-M.Proc.R.Soc. 258, 1-7 (1994).

9. Moritz, R. F. A. \& Hillesheim, E. Behav. Ecol. Sociobiol. 17, 87-98 (1985). 